\title{
Uncertain random programming models for fixed charge multi-item solid transportation problem
}

\author{
Hasan Dalman \\ Istanbul Gelisim University, Deparment of Computer Engineering, Avcilar, Istanbul, Turkey \\ Received: 28 September 2017, Accepted: 23 October 2017 \\ Published online: 3 February 2018.
}

\begin{abstract}
This paper presents uncertain random programming models for the fixed charge multiobjective multi-item solid transportation problem, which contains uncertain random variables for fixed charges, unit transportation costs, source, destination and conveyance constraints. Utilizing both uncertainty and randomness, the uncertain random programming model is first turned into an expected value programming model under chance constraints. Thus a deterministic model of the uncertain random model is obtained. Finally, numerical examples are given to illustrate the models.
\end{abstract}

Keywords: Uncertainty theory, uncertain random programming, transportation networks.

\section{Introduction}

The classical transportation problem (TP) consists of supply and demand constraints. But, in practice, decision makers are usually faced with more constraints such as goods constraint or mode constraints of carrying. In such conditions, TP is turned into a solid transportation problem (STP). The STP is first used by Shell [1] and then, Haley [2] presented a solution procedure for solving the STP.

The classical transportation problems in which different items are going to carry from diverse production points to diverse user points using various modes of conveyance are termed multi-item STPs.

Due to the lack of information, data deficiencies, complexity and unpredictable events, transportation systems are generally vague. To illustrate these types of impreciseness, the multi-item STPs are extensively studied under the fuzzy, stochastic and interval parameters [3-8].

It is admitted that a fundamental of probability theory is that the estimated probability distribution is near enough to the long-run cumulative frequency. Thus, there is no fear the parameters can be used as random variables when the adequate statistical data is present. Yet if there is no adequate statistical data, our fear is how to handle with the impreciseness of real world. Surely, taking into account the relevant parameters like fuzzy variables may be practicable, but it is inconvenient. Generally, decision-makers have no alternative aside form consult the field specialist to interpret the corresponding parameters. It indicates that the belief degree which depends seriously on the personal experiences. That is, the belief degree is an impreciseness, which is called as human uncertainty. For this, uncertainty theory was introduced by Liu [9] and refined by Liu [10] depend on normality, duality, subadditivity and product axioms. 
Since then, the uncertainty theory becomes a branch of mathematics. Also, uncertainty theory have studied both theoretically and practically in literature $[10,18]$.

TPs based on the uncertainty theory has been studied by some scholars. Cui and Sheng [19] gave an uncertain programming model for the STP. Zhang et al.[20] investigated uncertain fixed charge solid transportation problem, and Gao et al. [21] presented uncertain models on railway transportation planning problem. Dalman [22] introduced a multi-item STP with uncertain variables.

However, randomness and uncertainty usually consist complex systems. After the uncertain programming is presented in [23], Liu [24] introduced chance theory for representing such types of complex events including both uncertainty and randomness. Then some basic notations were given. After that, Liu [25] showed the operational law for calculating a monotone function of uncertain random variables and introduced the formula to calculate expected value. Uncertain random programming models have been investigated in literature [26]-[30] and so on.

According to my reading, no work has been given on uncertain random programming model for fixed charge multi-item solid transportation problems with uncertain random variables. Thus, this paper focuses on uncertain random programming for fixed charge multi-item solid transportation problem with uncertain random variables. Using the expected value of each objective function under the chance constraints, the model is transformed into a deterministic model. Finally, numerical examples are presented to illustrate the models.

This paper is constructed as follows: Section 2 presents some definitions and theorems about uncertainty and chance theory. Section 3 presents a definition for an uncertain random programming model. Based on uncertainty and randomness, the fixed charge multi-item STP model is modeled in Section 4. Numerical examples are given to illustrate different fixed charge multi-item STP model with some uncertain random variables in Section 5.

\section{Preliminaries}

\subsection{Uncertainty theory}

Basic definitions and notations of uncertainty theory are given here.

Definition 1. Let $\mathcal{L}$ be a $\sigma$-algebra on a nonempty set $\Gamma$. A set function $\mathcal{M}$ is called an uncertain measure if it satisfies the following axioms:

Axiom 1. (Normality Axiom) $\mathcal{M}\{\Gamma\}=1$;

Axiom 2. (Duality Axiom) $\mathcal{M}\{\Lambda\}+\mathcal{M}\left\{\Lambda^{c}\right\}=1$ for any $\Lambda \in \mathcal{L}$;

Axiom 3. (Subadditivity Axiom) For every countable sequence of $\left\{\Lambda_{i}\right\} \in \mathcal{L}$, we have

$$
\mathcal{M}\left\{\bigcup_{i=1}^{\infty} \Lambda_{i}\right\} \leq \sum_{i=1}^{\infty} \mathcal{M}\left\{\Lambda_{i}\right\} .
$$


The triplet $(\Gamma, \mathcal{L}, \mathcal{N})$ is called an uncertainty space, and each element $\Lambda$ in $\mathcal{L}$ is called an event. In addition, in order to obtain an uncertain measure of compound event, a product uncertain measure is defined by Liu [10] by the following product axiom:

Axiom 4. (Product Axiom) Let $\left(\Gamma_{k}, \mathcal{L}_{k}, \mathcal{M}_{k}\right)$ be uncertainty spaces for $k=1,2, \cdots$ The product uncertain measure $\mathcal{M}$ is an uncertain measure satisfying

$$
\mathcal{M}\left\{\prod_{k=1}^{\infty} \Lambda_{k}\right\}=\bigwedge_{k=1}^{\infty} \mathcal{M}_{k}\left\{\Lambda_{k}\right\}
$$

where $\Lambda_{k}$ are arbitrarily chosen events from $\mathcal{L}_{k}$ for $k=1,2, \cdots$, respectively.

Definition 2. (Liu [9]). An uncertain variable $\xi$ is a measurable function from an uncertainty space $(\Gamma, \mathcal{L}, \mathcal{M})$ to the set of real numbers, i.e., for any Borel set $B$ of real numbers, the set

$$
\{\xi \in B\}=\{\gamma \in \Gamma \mid \xi(\gamma) \in B\}
$$

is an event.

Definition 3. (Liu [9]). The uncertainty distribution $\Phi$ of an uncertain variable $\xi$ is defined by

$$
\Phi(x)=\mathcal{M}\{\xi \leq x\}, \quad \forall x \in \mathfrak{R} .
$$

Definition 4. (Liu[9]). Let $\xi$ be an uncertain variable. The expected value of $\xi$ is defined by

$$
E[\xi]=\int_{0}^{+\infty} \mathcal{M}\{\xi \geq r\} \mathrm{d} r-\int_{-\infty}^{0} \mathcal{M}\{\xi \leq r\} \mathrm{d} r
$$

provided that at least one of the above two integrals is finite. An uncertain variable $\xi$ is called linear if it has a linear uncertainty distribution

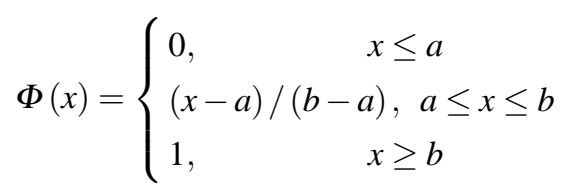

denoted by $t(a, b)$ where $a$ and $b$ are real numbers with $a<b$. Suppose that $\xi_{1}$ and $\xi_{2}$ are independent linear uncertain variables $€\left(a_{1}, b_{1}\right)$ and $€\left(a_{2}, b_{2}\right)$. Then the sum $\xi_{1}+\xi_{2}$ is also a linear uncertain variable $\measuredangle\left(a_{1}+a_{2}, b_{1}+b_{2}\right)$.

Definition 5. (Liu[31]). Let $\xi$ be an uncertain variable with a regular uncertainty distribution $\Phi(x)$. If the expected value is available, then

$$
E[\xi]=\int_{0}^{1} \Phi^{-1}(\alpha) \mathrm{d} \alpha
$$

where $\Phi^{-1}(\alpha)$ is the inverse uncertainty distribution of $\xi$.

Theorem 1. (Liu [31]). Assume $\xi_{1}, \xi_{2}, \cdots, \xi_{n}$ are independent uncertain variables with regular uncertainty distributions $\Phi_{1}, \Phi_{2}, \cdots, \Phi_{n}$, respectively. If the function $f\left(x_{1}, x_{2}, \cdots, x_{n}\right)$ is strictly increasing with respect to $x_{1}, x_{2}, \cdots, x_{m}$ and strictly 
decreasing with respect to $x_{m+1}, x_{m+2}, \cdots, x_{n}$, then $\xi=f\left(\xi_{1}, \xi_{2}, \cdots, \xi_{n}\right)$ has an inverse uncertainty distribution

$$
\Psi^{-1}(\alpha)=f\left(\Phi_{1}^{-1}(\alpha), \cdots, \Phi_{m}^{-1}(\alpha), \Phi_{m+1}^{-1}(1-\alpha), \cdots, \Phi_{n}^{-1}(1-\alpha)\right)
$$

In addition, Liu and Ha [32] shown that the uncertain variable $\xi$ has an expected value

$$
E[\xi]=\int_{0}^{1} f\left(\Phi_{1}^{-1}(\alpha), \cdots, \Phi_{m}^{-1}(\alpha), \Phi_{m+1}^{-1}(1-\alpha), \cdots, \Phi_{n}^{-1}(1-\alpha)\right) \mathrm{d} \alpha
$$

Theorem 2. (Liu[31]). Let $\xi$ and $\eta$ be independent uncertain variables with finite expected values. Since then, for any real numbers $a$ and $b$, we obtain

$$
\mathrm{E}[a \xi+b \eta]=a \mathrm{E}[\xi]+b \mathrm{E}[\eta]
$$

Theorem 3. (Liu[31]). Let $g\left(\left\{x, \xi_{1}, \xi_{2}, \ldots, \xi_{n}\right)\right.$ be constraint function. This function is strictly increasing with respect to $\xi_{1}, \xi_{2}, \ldots, \xi_{k}$ and strictly decreasing with respect to $\xi_{k+1} . \xi_{1}, \xi_{2}, \ldots, \xi_{k}$ are also independent uncertain variables with uncertain distributions $\Phi_{1}, \Phi_{2}, \ldots, \Phi_{n}$, respectively, then the chance constraint

$$
\mathscr{M}\left\{g\left(\left\{x, \xi_{1}, \xi_{2}, \ldots, \xi_{n}\right) \leq 0\right\} \geq \alpha\right.
$$

holds if and only if

$$
\begin{aligned}
g\left(\left\{x, \Phi_{1}^{-1}(\alpha), \ldots, \Phi_{k}^{-1}(\alpha),\right.\right. \\
\left.\quad \Phi_{k+1}^{-1}(1-\alpha), \ldots, \Phi_{n}^{-1}(1-\alpha)\right) \leq 0 .
\end{aligned}
$$

\subsection{Uncertain random variables}

Definition 6. An uncertain random variable is a measurable function $\xi$ from a chance space $(\Gamma, \mathcal{L}, \mathcal{M}) \times(\Omega, \mathcal{A}, \mathcal{P})$ to the set of real numbers such that $\{\xi \in \mathcal{B}\}$ is an event in $\mathcal{L} \times \mathcal{A}$ for any Borel set $\mathcal{B}$.

Liu Liu [24] verified that a chance measure meets normality, duality, and monotonicity properties. If an uncertain random variable turns to an uncertain variable, the chance distribution seems uncertainty distribution of an uncertain variable. Similarly, if an uncertain random variable turns to a random variable, the chance distribution seems probability distribution of a random variable.

Definition 7. Liu [24]. Let $(\Gamma, \mathcal{L}, \mathcal{M}) \times(\Omega, \mathcal{A}, \mathcal{P})$ be a chance space, and let $\Theta \in \mathcal{L} \times \mathcal{A}$. Then the chance measure of $\Theta$ is defned as

$$
C h\{\Theta\}=\int_{0}^{1} \operatorname{Pr}\{\omega \in \Omega \mid \mathrm{M}\{\gamma \in \Gamma(\gamma, \omega) \in \Theta\} \geq x\} d x
$$

Definition 8. Liu [24]. Let $\xi$ be an uncertain random variable. Then its chance distribution is defined by

$$
\Phi(x)=C h\{\xi \leq x\}
$$

for any real number $x$. 
Definition 9. Liu [24]. Let $\xi$ be an uncertain random variable. Then its expected value is defined by

$$
E[\xi]=\int_{0}^{\infty} \operatorname{Ch}\{\xi \geq x\} d r-\int_{-\infty}^{0} \operatorname{Ch}\{\xi \leq x\} d r
$$

provided that at least one of the two integrals is finite.

Definition 10. Liu [24]. Let $\xi$ be an uncertain random variable with regular chance distribution $\Phi$. Then

$$
E[\xi]=\int_{0}^{1} \Phi^{-1} \alpha d \alpha
$$

Theorem 4. Liu [25]. Let $\xi_{1}, \xi_{2}, \ldots, \xi_{n}$ be uncertain random variables on the chance space $(\Gamma, \mathcal{L}, \mathcal{M}) \times(\Omega, \mathcal{A}, \mathcal{P})$ and also let $f: R^{n} \rightarrow R$ be a measurable function. Thus $\xi=f\left(\xi_{1}, \xi_{2}, \ldots, \xi_{n}\right)$ an uncertain random variable determined by

$$
\xi(\gamma, \omega)=f\left(\xi_{1}\left(\gamma_{1}, \omega_{1}\right), \xi_{2}\left(\gamma_{2}, \omega_{2}\right), \ldots, \xi_{n}\left(\gamma_{n}, \omega_{n}\right)\right)
$$

for all $(\gamma, \omega) \in \Gamma \times \Omega$.

Theorem 5. Liu [25]. Let $\eta_{1}, \eta_{2}, \ldots, \eta_{m}$ be independent random variables with probability distributions $\Psi_{1}, \Psi_{2}, \ldots, \Psi_{m}$, respectively and let $\tau_{1}, \tau_{2}, \ldots, \tau_{n}$ be dependent and/or independent uncertain variables then the uncertain random variable

$$
\xi=f\left(\eta_{1}, \eta_{2}, \ldots, \eta_{m}, \tau_{1}, \tau_{2}, \ldots, \tau_{n}\right)
$$

has an expected value

$$
E[\xi]=\int_{R^{m}} E\left[f\left(y_{1}, y_{2}, \ldots, y_{m}, \tau_{1}, \tau_{2}, \ldots, \tau_{m}\right)\right] d \Psi_{1}\left(y_{1}\right), d \Psi_{2}\left(y_{2}\right), \ldots, d \Psi_{m}\left(y_{m}\right)
$$

where $E\left[f\left(y_{1}, y_{2}, \ldots, y_{m}, \tau_{1}, \tau_{2}, \ldots, \tau_{m}\right)\right]$ is the expected value of the uncertain variable $f\left(y_{1}, y_{2}, \ldots, y_{m}, \tau_{1}, \tau_{2}, \ldots, \tau_{m}\right)$ for any real numbers $y_{1}, y_{2}, \ldots, y_{m}$.

Theorem 6. Liu [25]. Assume $\eta_{1}$ and $\eta_{2}$ are random variables, $\tau_{1}$ and $\tau_{2}$ are independent uncertain variables and also $f_{1}$ and $f_{2}$ are measurable functions.Thus,

$$
E\left[f_{1}\left(\eta_{1}, \tau_{1}\right)+f_{2}\left(\eta_{2}, \tau_{2}\right)\right]=E\left[f_{1}\left(\eta_{1}, \tau_{1}\right)\right]+E\left[f_{2}\left(\eta_{2}, \tau_{2}\right)\right]
$$

\section{Uncertain random programming model for fixed charge multi-item solid transportation problem}

Suppose that $x=\left(x_{1}, x_{2}, \ldots, x_{n}\right)$ is $n$ - dimensioal decision vector, $\xi=\left(\xi_{1}, \xi_{2}, \ldots, \xi_{n}\right)$ is $n$-dimensional uncertain random vector, $f(x, \xi)$ and $g_{j}(x, \xi) \leq 0$ are the uncertan random objective and constraint functions, respectively. Taking confidence 
levels $\alpha=\left(\alpha_{1}, \alpha_{2}, \ldots, \alpha_{p}\right)$. Liu [25] formulated the following uncertain random programming model,

$$
\left\{\begin{array}{l}
\min _{x} E[f(x, \xi)], i=1,2, \ldots, q \\
\text { subject to } \\
\operatorname{Ch}\left\{g_{j}(x, \xi) \leq 0\right\} \geq \alpha_{j}, \quad j=1,2, \cdots, p
\end{array}\right.
$$

In a multi item STP there is a multi product to be carried from a set of origins to a set of destinations by a set of both similar or distinct conveyances. Every origin has such event to provide any of the destinations employing some of the conveyances and every destination can receive its demand from some of the origins employing some of the conveyances. Thus, every origin can provide zero, one or more destinations and the demand for each destination can be met by at least one origin. Each conveyance also is employed for zero, one or more unlocked ways from the origins to the destinations via conveyances. An unit cost is cosidered for carrying any quantity of products between the origins and the destinations via distinct conveyances. The purpose of the multi item STP is to minimize the total transportation cost by obtaining an optimal outcome of the products communicated in the unlocked directions by distinct conveyances.

Here the following notations are employed in all mathematical models of the multi item STP.

$\mathrm{M}:$ the number of origins,

$\mathrm{N}$ : the number of destinations,

$\mathrm{L}:$ the number of conveyances,

$\mathrm{R}:$ the number of items,

$i, j, k, p:$ the indexes used for source, destination and conveyance, respectively.

$a_{i}^{p}$ : the capacity of products of item $p$ at origins $i$

$b_{j}^{p}:$ the demand of products of item $p$ at destination $j$,

$e_{k}:$ the total transportation capacity of conveyance $k$,

$c_{i j k}^{p}$ is the unit cost of transporting one unit of item $p$ from source $i$ to destination $j$ by conveyance $k$,

$t_{i j k}^{p}$ is the fixed charge of carrying any amount of item $p$ from source $i$ to destination $j$ by conveyance $k$,

$x_{i j k}^{p}$ is the amount of item $p$ to be carried from source $i$ to destination $j$ by conveyance $k$.

$y_{i j k}^{p}$ is a binary variable, it takes value of 1 if the route from source $i$ to destination $j$ by conveyance $k$ is opened. This means $t_{i j k}^{p}>0$. That is, if it is decided to send an amount of product from source $i$ to destination $j$ by conveyance $k$ the value of $y_{i j k}^{p}$ is 1 . Otherwise it becomes 0 .

Under these notations, a mathematical model of multi item STP can be formulated as follows:

$$
\left\{\begin{array}{l}
f(x)=\min \sum_{p=1}^{R} \sum_{i=1}^{M} \sum_{j=1}^{N} \sum_{k=1}^{L} c_{i j k}^{p} x_{i j k}^{p}+\sum_{p=1}^{R} \sum_{i=1}^{M} \sum_{j=1}^{N} \sum_{k=1}^{L} t_{i j k}^{p} y_{i j k}^{p} \\
\text { s.t. }\left\{\begin{array}{l}
\sum_{j=1}^{N} \sum_{k=1}^{L} x_{i j k}^{p} \leq a_{i}^{p}, \forall i \in M ; \forall p \in R \\
\sum_{i=1}^{M} \sum_{k=1}^{L} x_{i j k}^{p} \geq b_{j}^{p}, \forall j \in N ; \\
\sum_{p=1}^{R} \sum_{i=1}^{M} \sum_{k=1}^{L} x_{i j k}^{p} \leq e_{k}, \forall k \in L \\
x_{i j k}^{p} \geq 0, \forall i \in M ; \forall j \in N ; \forall k \in L ; \forall p \in R \\
y_{i j k}^{p}=\left\{\begin{array}{l}
1 \text { if } x_{i j k}^{p}>0 \\
0 \text { if } x_{i j k}^{p}=0
\end{array}, \forall i \in M ; \forall j \in N ; \forall k \in L ; \forall p \in R\right.
\end{array}\right.
\end{array}\right.
$$


The objective function (a) minimizes the total transportation cost which is the sum of each unit cost. Constraint (b) assure that the whole amount of product $p$ carried from each origin to every destination should not be bigger than the capacity of that origin. Constraint (c) warrants that the demand for each destination should be replied. Constraint (d) shows the capacity of each conveyance constraint (e) portray non-negative variables,(f) represent binary variables, respectively.

We suppose that $x_{i j k}^{p}, y_{i j k}^{p}, a_{i}^{p}, b_{j}^{p}$, and $e_{k}$, are all uncertain random variables.Thus, we take them as uncertan random varables i.e., the per unit cost $\xi_{i j k}^{p}$, the fixed charge $\eta_{i j k}^{p}$, the capacity of each origin $\tilde{a}_{i}^{p}$, that of each destination $\tilde{b}_{j}^{p}$ and each conveyance $\tilde{b}_{j}^{p}$ are all uncertain random variables, respectively.

It means that $f(x, y ; \xi, \eta)$ is also uncertain random variables. Since $f$ has two uncertain random variables, it is hard to rank them easily. One way of doing this is to convert the uncertain random programming model into the expected value programming model.

Definition 11. A solution $x^{*}$ is referred to as the expected value of uncertain random fixed charge multi item solid transportation problem, if

$$
E\left[f\left(x^{*}, y^{*} ; \xi, \eta\right)\right] \leq E\left[f\left(x^{*}, y^{*} ; \xi, \eta\right)\right]
$$

holds for any feasible solution $x$.

Thus model(2) can be converted to its expected value programming model under chance constraints, as follows:

$$
\left\{\begin{array}{l}
\min \mathrm{E}\left[\sum_{p=1}^{R} \sum_{i=1}^{M} \sum_{j=1}^{N} \sum_{k=1}^{L} c_{i j k}^{p} x_{i j k}^{p}+\sum_{p=1}^{R} \sum_{i=1}^{M} \sum_{j=1}^{N} \sum_{k=1}^{L} t_{i j k}^{p} y_{i j k}^{p}\right] \\
\operatorname{Ch}\left\{\sum_{j=1}^{N} \sum_{k=1}^{L} x_{i j k}^{p} \leq a_{i}^{p}\right\} \geq \alpha_{i}^{p}, \forall i \in M ; \forall p \in R \\
\operatorname{Ch}\left\{\begin{array}{l}
\left.\sum_{i=1}^{M} \sum_{k=1}^{L} x_{i j k}^{p} \geq b_{j}^{p}\right\} \geq \beta_{j}^{p}, \forall j \in N ; \forall p \in R \\
C h\left\{\sum_{p=1}^{R} \sum_{i=1}^{M} \sum_{k=1}^{L} x_{i j k}^{p} \leq e_{k}\right\} \geq \gamma_{k}, \forall k \in L \\
x_{i j k}^{p} \geq 0, \forall i \in M ; \forall j \in N ; \forall k \in L ; \forall p \in R \\
y_{i j k}^{p}=\left\{\begin{array}{l}
1 \text { if } x_{i j k}^{p}>0 \\
0 \text { if } x_{i j k}^{p}=0
\end{array}, \forall i \in M ; \forall j \in N ; \forall k \in L ; \forall p \in R\right.
\end{array}\right.
\end{array}\right.
$$

where $\alpha_{i}^{p}, \beta_{j}^{p}, \gamma_{k}$ are preconcerted confidence level for $\forall i \in M ; \forall i \in N ; \forall k \in M ; \forall k \in L ; \forall p \in R$.

In this model, the given parameters are either uncertain and random variables. if $a_{i}^{p}, b_{j}^{p}, e_{k}$ are uncertain variables, the change measure degenerates and becomes the uncertainty measure. At the same time, if $a_{i}^{p}, b_{j}^{p}, e_{k}$ are random variables, the change measure degenerates and becomes the probability measure.

Let us assume that the $c_{i j k}^{p}$ is $\left(c_{i j k}^{p}\right)^{\prime}$ for uncetain variables and $\left(c_{i j k}^{p}\right)^{\prime \prime}$ for random variables. Similarly, $t_{i j k}^{p}$ is $\left(t_{i j k}^{p}\right)^{\prime}$ for uncetain variables and $\left(t_{i j k}^{p}\right)^{\prime \prime}$ for random variables. Moreover, $a_{i}^{p}, b_{j}^{p}, e_{k}$ are $\left(a_{i}^{p}\right)^{\prime},\left(b_{j}^{p}\right)^{\prime},\left(e_{k}\right)^{\prime}$ for uncertain variables and $\left(a_{i}^{p}\right)^{\prime \prime},\left(b_{j}^{p}\right)^{\prime \prime},\left(e_{k}\right)^{\prime \prime}$ for random variables. 
Then model (3) can be transformed into an equivalent form as follows:

$$
\begin{aligned}
& \min \mathrm{E}\left[\begin{array}{c}
\sum_{p=1}^{R} \sum_{i=1}^{M} \sum_{j=1}^{N} \sum_{k=1}^{L}\left(c_{i j k}^{p}\right)^{\prime} x_{i j k}^{p}+\sum_{p=1}^{R} \sum_{i=1}^{M} \sum_{j=1}^{N} \sum_{k=1}^{L}\left(c_{i j k}^{p}\right)^{\prime \prime} x_{i j k}^{p}+\sum_{p=1}^{R} \sum_{i=1}^{M} \sum_{j=1}^{N} \sum_{k=1}^{L}\left(t_{i j k}^{p}\right)^{\prime} y_{i j k}^{p} \\
+\sum_{p=1}^{R} \sum_{i=1}^{M} \sum_{j=1}^{N} \sum_{k=1}^{L}\left(t_{i j k}^{p}\right)^{\prime \prime} y_{i j k}^{p}
\end{array}\right]
\end{aligned}
$$

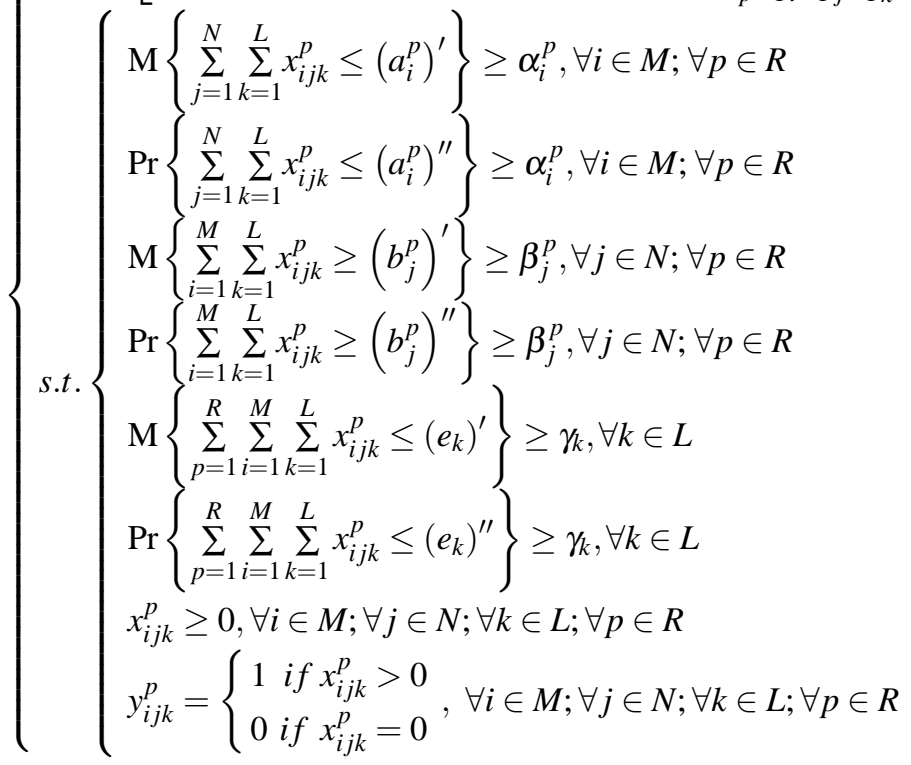

Lemma 1. Suppose $\left(a_{i}^{p}\right)^{\prime},\left(b_{j}^{p}\right)^{\prime},\left(e_{k}\right)^{\prime},\left(c_{i j k}^{p}\right)^{\prime},\left(t_{i j k}^{p}\right)^{\prime}$ independent uncertain vaiables with regular uncertainty distributions $\Phi_{\left(a_{i}^{p}\right)^{\prime}}, \Phi_{\left(b_{j}^{p}\right)^{\prime}}, \Phi_{\left(e_{k}\right)^{\prime}}, \Phi_{\left(c_{i j k}^{p}\right)^{\prime}}, \Phi_{\left(t_{i j k}^{p}\right)^{\prime}}$, respectively. And also suppose $\left(a_{i}^{p}\right)^{\prime},\left(b_{j}^{p}\right)^{\prime},\left(e_{k}\right)^{\prime},\left(c_{i j k}^{p}\right)^{\prime},\left(t_{i j k}^{p}\right)^{\prime}$ are independent random vaiables with regular probability distributions $\Psi_{\left(a_{i}^{p}\right)^{\prime}}, \Psi_{\left(b_{j}^{p}\right)^{\prime}}, \Psi_{\left(e_{k}\right)^{\prime}}, \Psi_{\left(c_{i j k}^{p}\right)^{\prime}}, \Psi_{\left(t_{i j k}^{p}\right)^{\prime}}$. Thus, model (4) converts into the following conventional mathematical programming model.

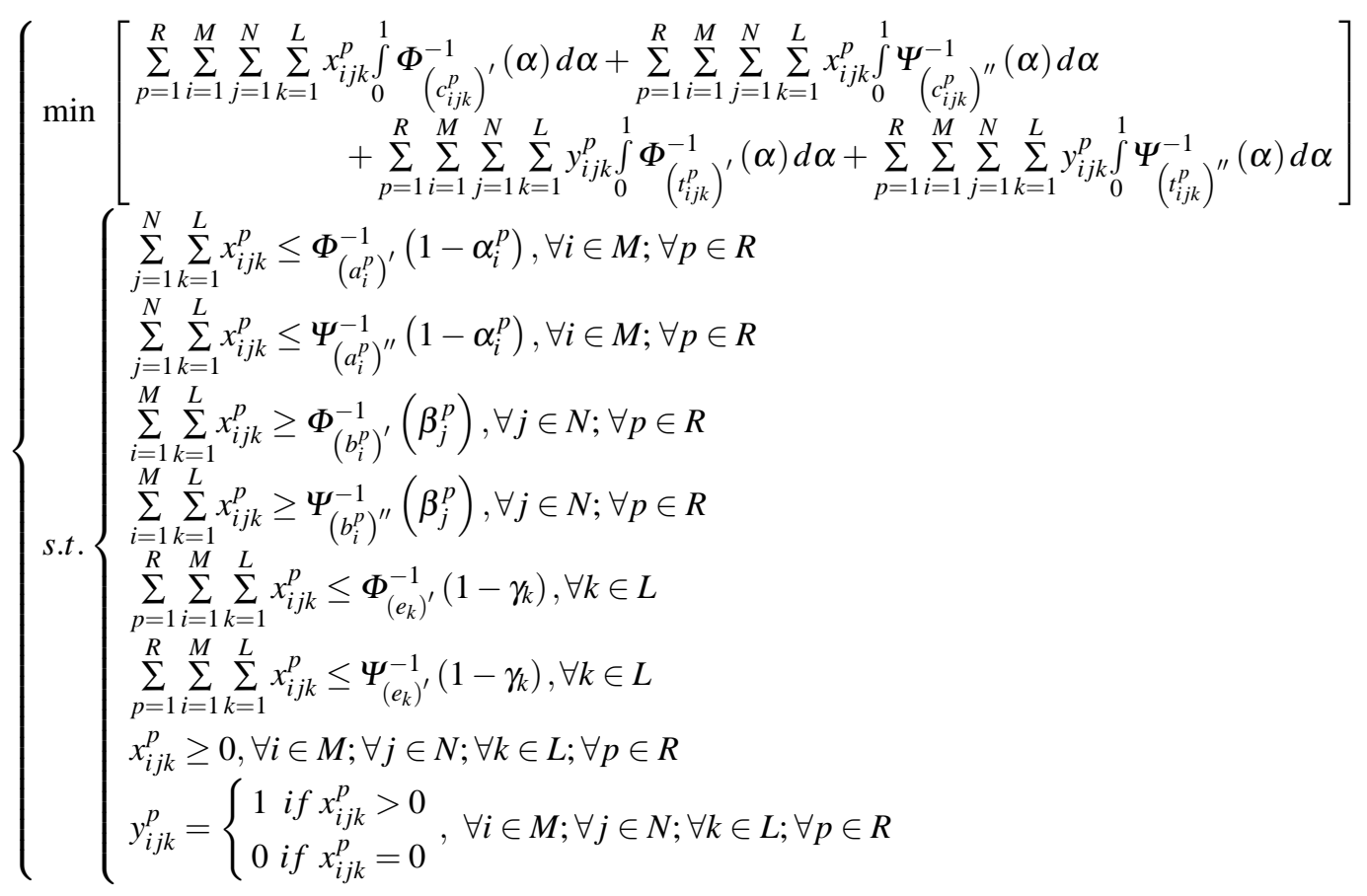


Proof. From Theorem 6 and linear property of the expected value of uncertain random variables, we obtain

$$
\begin{aligned}
& \mathrm{E}\left[\begin{array}{c}
\sum_{p=1}^{R} \sum_{i=1}^{M} \sum_{j=1}^{N} \sum_{k=1}^{L}\left(c_{i j k}^{p}\right)^{\prime} x_{i j k}^{p}+\sum_{p=1}^{R} \sum_{i=1}^{M} \sum_{j=1}^{N} \sum_{k=1}^{L}\left(c_{i j k}^{p}\right)^{\prime \prime} x_{i j k}^{p} \\
+\sum_{p=1}^{R} \sum_{i=1}^{M} \sum_{j=1}^{N} \sum_{k=1}^{L}\left(t_{i j k}^{p}\right)^{\prime} y_{i j k}^{p}+\sum_{p=1}^{R} \sum_{i=1}^{M} \sum_{j=1}^{N} \sum_{k=1}^{L}\left(t_{i j k}^{p}\right)^{\prime \prime} y_{i j k}^{p}
\end{array}\right] \\
& =\left\{\begin{aligned}
\mathrm{E}\left[\sum_{p=1}^{R} \sum_{i=1}^{M} \sum_{j=1}^{N} \sum_{k=1}^{L}\left(c_{i j k}^{p}\right)^{\prime} x_{i j k}^{p}\right]+\mathrm{E}\left[\sum_{p=1}^{R} \sum_{i=1}^{M} \sum_{j=1}^{N} \sum_{k=1}^{L}\left(c_{i j k}^{p}\right)^{\prime \prime} x_{i j k}^{p}\right] \\
+\mathrm{E}\left[\sum_{p=1}^{R} \sum_{i=1}^{M} \sum_{j=1}^{N} \sum_{k=1}^{L}\left(t_{i j k}^{p}\right)^{\prime} y_{i j k}^{p}\right]+\mathrm{E}\left[\sum_{p=1}^{R} \sum_{i=1}^{M} \sum_{j=1}^{N} \sum_{k=1}^{L}\left(t_{i j k}^{p}\right)^{\prime \prime} y_{i j k}^{p}\right]
\end{aligned}\right. \\
& =\left\{\begin{aligned}
\sum_{p=1}^{R} \sum_{i=1}^{M} \sum_{j=1}^{N} \sum_{k=1}^{L} \mathrm{E}\left[\left(c_{i j k}^{p}\right)^{\prime}\right] x_{i j k}^{p}+\sum_{p=1}^{R} \sum_{i=1}^{M} \sum_{j=1}^{N} \sum_{k=1}^{L} \mathrm{E}\left[\left(c_{i j k}^{p}\right)^{\prime \prime}\right] x_{i j k}^{p} \\
+\sum_{p=1}^{R} \sum_{i=1}^{M} \sum_{j=1}^{N} \sum_{k=1}^{L} \mathrm{E}\left[\left(t_{i j k}^{p}\right)^{\prime}\right] y_{i j k}^{p}+\sum_{p=1}^{R} \sum_{i=1}^{M} \sum_{j=1}^{N} \sum_{k=1}^{L} \mathrm{E}\left[\left(t_{i j k}^{p}\right)^{\prime \prime}\right] y_{i j k}^{p}
\end{aligned}\right. \\
& =\left\{\begin{aligned}
\sum_{p=1}^{R} \sum_{i=1}^{M} \sum_{j=1}^{N} \sum_{k=1}^{L} \int_{0}^{1} \Phi_{\left(c_{i j k}^{p}\right)^{\prime}}^{-1}(\alpha) d \alpha+\sum_{p=1}^{R} \sum_{i=1}^{M} \sum_{j=1}^{N} \sum_{k=1}^{L} \int_{0}^{1} \Psi_{\left(c_{i j k}^{p}\right)^{\prime \prime}}^{-1}(\alpha) d \alpha \\
+\sum_{p=1}^{R} \sum_{i=1}^{M} \sum_{j=1}^{N} \sum_{k=1}^{L} \int_{0}^{1} \Phi_{\left(t_{i j k}^{p}\right)^{\prime}}^{-1}(\alpha) d \alpha+\sum_{p=1}^{R} \sum_{i=1}^{M} \sum_{j=1}^{N} \sum_{k=1}^{L} \int_{0}^{1} \Psi_{\left(t_{i j k}^{p}\right)^{-1}}^{\prime \prime}(\alpha) d \alpha
\end{aligned}\right.
\end{aligned}
$$

From Theorem 3, the first uncertain constraints

$$
\mathbf{M}\left\{\sum_{j=1}^{N} \sum_{k=1}^{L} x_{i j k}^{p} \leq\left(a_{i}^{p}\right)^{\prime}\right\} \geq \alpha_{i}^{p}, \forall i \in M ; \forall p \in R
$$

are equivalent to the constraints

$$
\sum_{j=1}^{N} \sum_{k=1}^{L} x_{i j k}^{p} \leq \Phi_{\left(a_{i}^{p}\right)^{\prime}}^{-1}\left(1-\alpha_{i}^{p}\right), \forall i \in M ; \forall p \in R
$$

Similarly, other constraints $\mathrm{M}\left\{\sum_{i=1}^{M} \sum_{k=1}^{L} x_{i j k}^{p} \geq\left(b_{j}^{p}\right)^{\prime}\right\} \geq \beta_{j}^{p}, \forall j \in N ; \forall p \in R ; \mathrm{M}\left\{\sum_{p=1}^{R} \sum_{i=1}^{M} \sum_{k=1}^{L} x_{i j k}^{p} \leq\left(e_{k}\right)^{\prime}\right\} \geq \gamma_{k}, \forall k \in L$ are equivalent to the inequalities $\sum_{i=1}^{M} \sum_{k=1}^{L} x_{i j k}^{p} \geq \Phi_{\left(b_{i}^{p}\right)^{\prime}}^{-1}\left(\beta_{j}^{p}\right), \forall j \in N ; \forall p \in R ; \sum_{p=1}^{R} \sum_{i=1}^{M} \sum_{k=1}^{L} x_{i j k}^{p} \leq \Phi_{\left(e_{k}\right)^{\prime}}^{-1}\left(1-\gamma_{k}\right), \forall k \in L$, respectively. However, the first probabilistic constraints

$$
\operatorname{Pr}\left\{\sum_{j=1}^{N} \sum_{k=1}^{L} x_{i j k}^{p} \leq\left(a_{i}^{p}\right)^{\prime \prime}\right\} \geq \alpha_{i}^{p}, \forall i \in M ; \forall p \in R
$$

can be written as:

$$
\operatorname{Pr}\left\{\left(a_{i}^{p}\right)^{\prime \prime} \geq \sum_{j=1}^{N} \sum_{k=1}^{L} x_{i j k}^{p}\right\} \geq \alpha_{i}^{p}, \forall i \in M ; \forall p \in R
$$

Then the following inequality can be obtained easily.

$$
1-\Psi\left\{\sum_{j=1}^{N} \sum_{k=1}^{L} x_{i j k}^{p}\right\} \geq \alpha_{i}^{p}
$$


That is,

$$
\Psi\left\{\sum_{j=1}^{N} \sum_{k=1}^{L} x_{i j k}^{p}\right\} \leq 1-\alpha_{i}^{p}
$$

Thus,

$$
\operatorname{Pr}\left\{\sum_{j=1}^{N} \sum_{k=1}^{L} x_{i j k}^{p} \leq\left(a_{i}^{p}\right)^{\prime \prime}\right\} \geq \alpha_{i}^{p}
$$

are equivalent to the inequalities

$$
\sum_{j=1}^{N} \sum_{k=1}^{L} x_{i j k}^{p} \leq \Psi_{\left(a_{i}^{p}\right)^{\prime \prime}}^{-1}\left(1-\alpha_{i}^{p}\right)
$$

Other constraints can be proven in this way. So the proof is verify.

\section{Numerical examples}

Example 1. Suppose that $\left(a_{i}^{p}\right)^{\prime},\left(b_{j}^{p}\right)^{\prime},\left(e_{k}\right)^{\prime},\left(c_{i j k}^{p}\right)^{\prime},\left(t_{i j k}^{p}\right)^{\prime}$ are independent uncertain linear vaiables

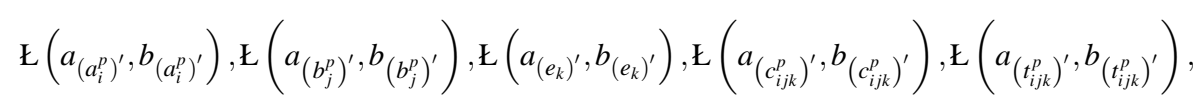

and also $\left(a_{i}^{p}\right)^{\prime \prime},\left(b_{j}^{p}\right)^{\prime \prime},\left(e_{k}\right)^{\prime \prime},\left(c_{i j k}^{p}\right)^{\prime \prime},\left(t_{i j k}^{p}\right)^{\prime \prime}$ are the normal probability variables

$$
\mathcal{N}\left(\mu_{\left(a_{i}^{p}\right)^{\prime \prime}}, \sigma_{\left(a_{i}^{p}\right)^{\prime \prime}}\right), \mathcal{N}\left(\mu_{\left(b_{j}^{p}\right)^{\prime \prime}}, \sigma_{\left(b_{j}^{p}\right)^{\prime \prime}}\right), \mathcal{N}\left(\mu_{\left(e_{k}\right)^{\prime \prime}}, \sigma_{\left(e_{k}\right)^{\prime \prime}}\right), \mathcal{N}\left(\mu_{\left(c_{i j k}^{p}\right)^{\prime \prime}}, \sigma_{\left(c_{i j k}^{p}\right)^{\prime \prime}}\right), \mathcal{N}\left(\mu_{\left(t_{i j k}^{p}\right)^{\prime \prime}}, \sigma_{\left(t_{i j k}^{p}\right)^{\prime \prime}}\right)
$$

Thus, model (5) transforms into the following equivalent deterministic single objective programming model.

$$
\begin{aligned}
& \min \left[\begin{array}{c}
\sum_{p=1}^{R} \sum_{i=1}^{M} \sum_{j=1}^{N} \sum_{k=1}^{L} \frac{\left(a_{\left.\left(c_{i j k}^{p}\right)^{\prime+b}\left(c_{i j k}^{p}\right)^{\prime}\right)}\right.}{2} x_{i j k}^{p}+\sum_{p=1}^{R} \sum_{i=1}^{M} \sum_{j=1}^{N} \sum_{k=1}^{L} \mu_{\left(c_{i j k}^{p}\right)^{\prime \prime}} x_{i j k}^{p} \\
+\sum_{p=1}^{R} \sum_{i=1}^{M} \sum_{j=1}^{N} \sum_{k=1}^{L} \frac{\left(a_{\left.\left(t_{i j k}^{p}\right)^{\prime+b}\left(t_{i j k}^{p}\right)^{\prime}\right)}\right.}{2} y_{i j k}^{p}+\sum_{p=1}^{R} \sum_{i=1}^{M} \sum_{j=1}^{N} \sum_{k=1}^{L} \mu_{\left(t_{i j k}^{p}\right)^{\prime \prime}} y_{i j k}^{p}
\end{array}\right] \\
& s . t .\left\{\begin{array}{l}
\sum_{j=1}^{N} \sum_{k=1}^{L} x_{i j k}^{p} \leq \alpha_{i}^{p} a_{\left(a_{i}^{p}\right)^{\prime}}+\left(1-\alpha_{i}^{p}\right) b_{\left(a_{i}^{p}\right)^{\prime}}, \forall i \in M ; \forall p \in R \\
\sum_{j=1}^{N} \sum_{k=1}^{L} x_{i j k}^{p} \leq \mu_{\left(a_{i}^{p}\right)^{\prime \prime}}+\sigma_{\left(a_{i}^{p}\right)^{\prime \prime}}\left(1-\alpha_{i}^{p}\right), \forall i \in M ; \forall p \in R \\
\sum_{i=1}^{M} \sum_{k=1}^{L} x_{i j k}^{p} \geq a_{\left(b_{i}^{p}\right)^{\prime}}\left(1-\beta_{j}^{p}\right)+b_{\left(b_{i}^{p}\right)^{\prime}} \beta_{j}^{p}, \forall j \in N ; \forall p \in R \\
\sum_{i=1}^{M} \sum_{k=1}^{L} x_{i j k}^{p} \geq \mu_{\left(b_{i}^{p}\right)^{\prime \prime}}+\sigma_{\left(b_{i}^{p}\right)^{\prime \prime}} \beta_{j}^{p}, \forall j \in N ; \forall p \in R \\
\sum_{p=1}^{R} \sum_{i=1}^{M} \sum_{k=1}^{L} x_{i j k}^{p} \leq a_{\left(e_{k}\right)^{\prime}}\left(1-\gamma_{k}\right)+b_{\left(e_{k}\right)^{\prime}} \gamma_{k}, \forall k \in L \\
\sum_{p=1}^{R} \sum_{i=1}^{M} \sum_{k=1}^{L} x_{i j k}^{p} \leq \mu_{\left(e_{k}\right)^{\prime \prime}}+\sigma_{\left(e_{k}\right)^{\prime \prime}}\left(1-\gamma_{k}\right), \forall k \in L \\
x_{i j k}^{p} \geq 0, \forall i \in M ; \forall j \in N ; \forall k \in L ; \forall p \in R \\
y_{i j k}^{p}=\left\{\begin{array}{l}
1 \text { if } x_{i j k}^{p}>0 \\
0 \text { if } x_{i j k}^{p}=0
\end{array}, \forall i \in M ; \forall j \in N ; \forall k \in L ; \forall p \in R\right.
\end{array}\right.
\end{aligned}
$$


NTMSCI 6, No. 1, 37-51 (2018) / www.ntmsci.com

BISK

47

Example 2. Suppose $\left(a_{i}^{p}\right)^{\prime},\left(b_{j}^{p}\right)^{\prime},\left(e_{k}\right)^{\prime},\left(c_{i j k}^{p}\right)^{\prime},\left(t_{i j k}^{p}\right)^{\prime}$ are independent uncertain zigzag variables

$$
\mathrm{Z}\left(a_{\left(a_{i}^{p}\right)^{\prime}}, b_{\left(a_{i}^{p}\right)^{\prime}} d_{\left(a_{i}^{p}\right)^{\prime}}\right), \mathrm{Z}\left(a_{\left(b_{j}^{p}\right)^{\prime}}, b_{\left(b_{j}^{p}\right)^{\prime}} d_{\left(b_{j}^{p}\right)^{\prime}}\right), \mathrm{Z}\left(a_{\left(e_{k}\right)^{\prime}}, b_{\left(e_{k}\right)^{\prime}} d_{\left(e_{k}\right)^{\prime}}, \mathrm{Z}\left(a_{\left(c_{i j k}^{p}\right)^{\prime}}, b_{\left(c_{i j k}^{p}\right)^{\prime}} d_{\left(c_{i j k}^{p}\right)^{\prime}}\right), \mathrm{Z}\left(a_{\left(t_{i j k}^{p}\right)^{\prime}}, b_{\left(t_{i j k}^{p}\right)^{\prime}} d_{\left(t_{i j k}^{p}\right)^{\prime}}\right),\right.
$$

and also $\left(a_{i}^{p}\right)^{\prime \prime},\left(b_{j}^{p}\right)^{\prime \prime},\left(e_{k}\right)^{\prime \prime},\left(c_{i j k}^{p}\right)^{\prime \prime},\left(t_{i j k}^{p}\right)^{\prime \prime}$ are the normal probability variables

$$
\mathcal{N}\left(\mu_{\left(a_{i}^{p}\right)^{\prime \prime}}, \sigma_{\left(a_{i}^{p}\right)^{\prime \prime}}\right), \mathcal{N}\left(\mu_{\left(b_{j}^{p}\right)^{\prime \prime}}, \sigma_{\left(b_{j}^{p}\right)^{\prime \prime}}\right), \mathcal{N}\left(\mu_{\left(e_{k}\right)^{\prime \prime}}, \sigma_{\left(e_{k}\right)^{\prime \prime}}\right), \mathcal{N}\left(\mu_{\left(c_{i j k}^{p}\right)^{\prime \prime}}, \sigma_{\left(c_{i j k}^{p}\right)^{\prime \prime}}\right), \mathcal{N}\left(\mu_{\left(t_{i j k}^{p}\right)^{\prime \prime}}, \sigma_{\left(t_{i j k}^{p}\right)^{\prime \prime}}\right)
$$

respectively. Let confidence levels $\alpha_{i}^{p}, \beta_{j}^{p}, \gamma_{k}$ be greater than 0.5 . Then, model (5) transforms into the following equivalent deterministic single objective programming model.

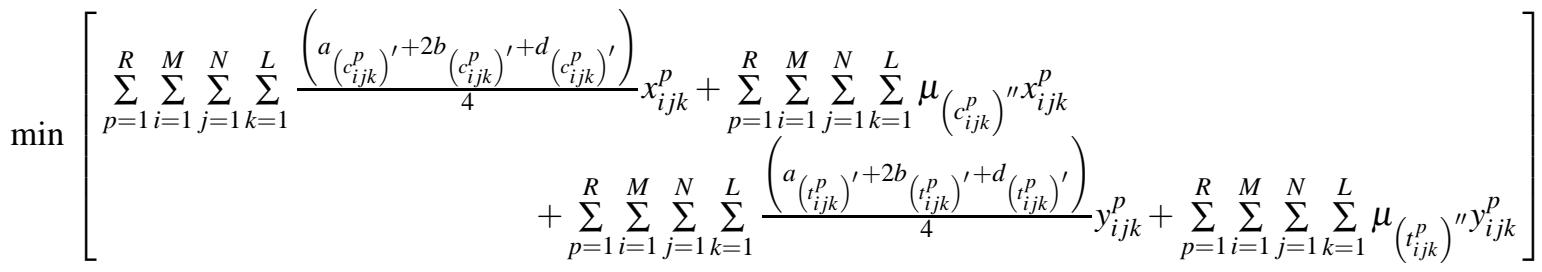

$$
\begin{aligned}
& \left\{\begin{array}{l}
\sum_{j=1}^{N} \sum_{k=1}^{L} x_{i j k}^{p} \leq\left(2 \alpha_{i}^{p}-1\right) a_{\left(a_{i}^{p}\right)^{\prime}}+2\left(1-\alpha_{i}^{p}\right) b_{\left(a_{i}^{p}\right)^{\prime}}, \forall i \in M ; \forall p \in R \\
\sum_{j=1}^{N} \sum_{k=1}^{L} x_{i j k}^{p} \leq \mu_{\left(a_{i}^{p}\right)^{\prime \prime}}+\sigma_{\left(a_{i}^{p}\right)^{\prime \prime}}\left(1-\alpha_{i}^{p}\right), \forall i \in M ; \forall p \in R \\
\sum_{i=1}^{M} \sum_{k=1}^{L} x_{i j k}^{p} \geq a_{\left(b_{i}^{p}\right)^{\prime}}\left(2-2 \beta_{j}^{p}\right)+b_{\left(b_{i}^{p}\right)^{\prime}}\left(2 \beta_{j}^{p}-1\right), \forall j \in N ; \forall p \in R \\
\sum_{i=1}^{M} \sum_{k=1}^{L} x_{i j k}^{p} \geq \mu_{\left(b_{i}^{p}\right)^{\prime \prime}}+\sigma_{\left(b_{i}^{p}\right)^{\prime \prime}} \beta_{j}^{p}, \forall j \in N ; \forall p \in R \\
\sum_{p=1}^{R} \sum_{i=1}^{M} \sum_{k=1}^{L} x_{i j k}^{p} \leq a_{\left(e_{k}\right)^{\prime}}\left(2 \gamma_{k}-1\right)+2 b_{\left(e_{k}\right)^{\prime}}\left(1-\gamma_{k}\right), \forall k \in L \\
\sum_{p=1}^{R} \sum_{i=1}^{M} \sum_{k=1}^{L} x_{i j k}^{p} \leq \mu_{\left(e_{k}\right)^{\prime \prime}}+\sigma_{\left(e_{k}\right)^{\prime \prime}}\left(1-\gamma_{k}\right), \forall k \in L \\
x_{i j k}^{p} \geq 0, \forall i \in M ; \forall j \in N ; \forall k \in L ; \forall p \in R \\
y_{i j k}^{p}=\left\{\begin{array}{l}
1 \text { if } x_{i j k}^{p}>0 \\
0 \text { if } x_{i j k}^{p}=0
\end{array}, \forall i \in M ; \forall j \in N ; \forall k \in L ; \forall p \in R\right.
\end{array}\right.
\end{aligned}
$$

Example 3. Suppose $\left(a_{i}^{p}\right)^{\prime},\left(b_{j}^{p}\right)^{\prime},\left(e_{k}\right)^{\prime},\left(c_{i j k}^{p}\right)^{\prime},\left(t_{i j k}^{p}\right)^{\prime}$ are independent uncertain normal variables

$$
\mathcal{N}\left(e_{\left(a_{i}^{p}\right)^{\prime}}, \sigma_{\left(a_{i}^{p}\right)^{\prime}}\right), \mathcal{N}\left(e_{\left(b_{j}^{p}\right)^{\prime}}, \sigma_{\left(b_{j}^{p}\right)^{\prime}}\right), \mathcal{N}\left(e_{\left(e_{k}\right)^{\prime}}, \sigma_{\left(e_{k}\right)^{\prime}}\right), \mathcal{N}\left(e_{\left(c_{i j k}^{p}\right)^{\prime}}, \sigma_{\left(c_{i j k}^{p}\right)^{\prime}}\right), \mathcal{N}\left(e_{\left(t_{i j k}^{p}\right)^{\prime}}, \sigma_{\left(t_{i j k}^{p}\right)^{\prime}}\right)
$$

and also $\left(a_{i}^{p}\right)^{\prime \prime},\left(b_{j}^{p}\right)^{\prime \prime},\left(e_{k}\right)^{\prime \prime},\left(c_{i j k}^{p}\right)^{\prime \prime},\left(t_{i j k}^{p}\right)^{\prime \prime}$ are the normal probability variables

$$
\mathcal{N}\left(\mu_{\left(a_{i}^{p}\right)^{\prime \prime}}, \sigma_{\left(a_{i}^{p}\right)^{\prime \prime}}\right), \mathcal{N}\left(\mu_{\left(b_{j}^{p}\right)^{\prime \prime}}, \sigma_{\left(b_{j}^{p}\right)^{\prime \prime}}\right), \mathcal{N}\left(\mu_{\left(e_{k}\right)^{\prime \prime}}, \sigma_{\left(e_{k}\right)^{\prime \prime}}, \mathcal{N}\left(\mu_{\left(c_{i j k}^{p}\right)^{\prime \prime}}, \sigma_{\left(c_{i j k}^{p}\right)^{\prime \prime}}\right), \mathcal{N}\left(\mu_{\left(t_{i j k}^{p}\right)^{\prime \prime}}, \sigma_{\left(t_{i j k}^{p}\right)^{\prime \prime}}\right),\right.
$$

(c) 2018 BISKA Bilisim Technology 
Thus, model (5) transforms into the following equivalent deterministic single objective programming model.

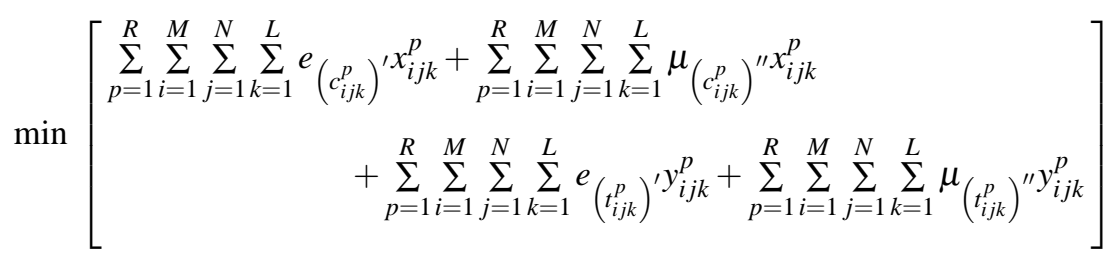

$$
\begin{aligned}
& \left\{\begin{array}{l}
\sum_{j=1}^{N} \sum_{k=1}^{L} x_{i j k}^{p} \leq e_{\left(a_{i}^{p}\right)^{\prime}}+\frac{\sqrt{3} \sigma_{\left(a_{i}^{p}\right)^{\prime}}}{\pi} \ln \frac{1-\alpha_{i}^{p}}{\alpha_{i}^{p}}, \forall i \in M ; \forall p \in R \\
\sum_{j=1}^{N} \sum_{k=1}^{L} x_{i j k}^{p} \leq \mu_{\left(a_{i}^{p}\right)^{\prime \prime}}+\sigma_{\left(a_{i}^{p}\right)^{\prime \prime}}\left(1-\alpha_{i}^{p}\right), \forall i \in M ; \forall p \in R \\
\sum_{i=1}^{M} \sum_{k=1}^{L} x_{i j k}^{p} \geq e_{\left(b_{i}^{p}\right)^{\prime}}+\frac{\sqrt{3} \sigma_{\left(b_{i}^{p}\right)^{\prime}}}{\pi} \ln \frac{\beta_{j}^{p}}{1-\beta_{j}^{p}}, \forall j \in N ; \forall p \in R
\end{array}\right. \\
& \text { s.t. }\left\{\begin{array}{l}
\sum_{i=1}^{M} \sum_{k=1}^{L} x_{i j k}^{p} \geq \mu_{\left(b_{i}^{p}\right)^{\prime \prime}}+\sigma_{\left(b_{i}^{p}\right)^{\prime \prime}} \beta_{j}^{p}, \forall j \in N ; \forall p \in R \\
\sum_{p=1}^{R} \sum_{i=1}^{M} \sum_{k=1}^{L} x_{i j k}^{p} \leq e_{\left(e_{k}\right)^{\prime}}+\frac{\sqrt{3} \sigma_{\left(e_{i}^{p}\right)^{\prime}}}{\pi} \ln \frac{1-\gamma_{k}}{\gamma_{k}}, \forall k \in L \\
\sum_{p=1}^{R} \sum_{i=1}^{M} \sum_{k=1}^{L} x_{i j k}^{p} \leq \mu_{\left(e_{k}\right)^{\prime \prime}}+\sigma_{\left(e_{k}\right)^{\prime \prime}}\left(1-\gamma_{k}\right), \forall k \in L \\
x_{i j k}^{p} \geq 0, \forall i \in M ; \forall j \in N ; \forall k \in L ; \forall p \in R \\
y_{i j k}^{p}=\left\{\begin{array}{l}
1 \text { if } x_{i j k}^{p}>0 \\
0 \text { if } x_{i j k}^{p}=0
\end{array}, \forall i \in M ; \forall j \in N ; \forall k \in L ; \forall p \in R\right.
\end{array}\right.
\end{aligned}
$$

Example 4. Suppose $\left(a_{i}^{p}\right)^{\prime},\left(b_{j}^{p}\right)^{\prime},\left(e_{k}\right)^{\prime},\left(c_{i j k}^{p}\right)^{\prime},\left(t_{i j k}^{p}\right)^{\prime}$ are independent lognormal uncertain variables

$$
\mathcal{N}\left(e_{\left(a_{i}^{p}\right)^{\prime}}, \sigma_{\left(a_{i}^{p}\right)^{\prime}}\right), \mathcal{N}\left(e_{\left(b_{j}^{p}\right)^{\prime}}, \sigma_{\left(b_{j}^{p}\right)^{\prime}}\right), \mathcal{N}\left(e_{\left(e_{k}\right)^{\prime}}, \sigma_{\left(e_{k}\right)^{\prime}}\right), \mathcal{N}\left(e_{\left(c_{i j k}^{p}\right)^{\prime}}, \sigma_{\left(c_{i j k}^{p}\right)^{\prime}}\right), \mathcal{N}\left(e_{\left(t_{i j k}^{p}\right)^{\prime}}, \sigma_{\left(t_{i j k}^{p}\right)^{\prime}}\right)
$$

and also $\left(a_{i}^{p}\right)^{\prime \prime},\left(b_{j}^{p}\right)^{\prime \prime},\left(e_{k}\right)^{\prime \prime},\left(c_{i j k}^{p}\right)^{\prime \prime},\left(t_{i j k}^{p}\right)^{\prime \prime}$ are the normal probability variables

$$
\mathcal{N}\left(\mu_{\left(a_{i}^{p}\right)^{\prime \prime}}, \sigma_{\left(a_{i}^{p}\right)^{\prime \prime}}\right), \mathcal{N}\left(\mu_{\left(b_{j}^{p}\right)^{\prime \prime}}, \sigma_{\left(b_{j}^{p}\right)^{\prime \prime}}\right), \mathcal{N}\left(\mu_{\left(e_{k}\right)^{\prime \prime}}, \sigma_{\left(e_{k}\right)^{\prime \prime}}\right), \mathcal{N}\left(\mu_{\left(c_{i j k}^{p}\right)^{\prime \prime}}, \sigma_{\left(c_{i j k}^{p}\right)^{\prime \prime}}\right), \mathcal{N}\left(\mu_{\left(t_{i j k}^{p}\right)^{\prime \prime}}, \sigma_{\left(t_{i j k}^{p}\right)^{\prime \prime}}\right)
$$

respectively. Moreover, let $\left(c_{i j k}^{p}\right)^{\prime},\left(t_{i j k}^{p}\right)^{\prime}$ be less than $\pi / 3$. Then, model (5) transforms into the following equivalent deterministic single objective programming model. 


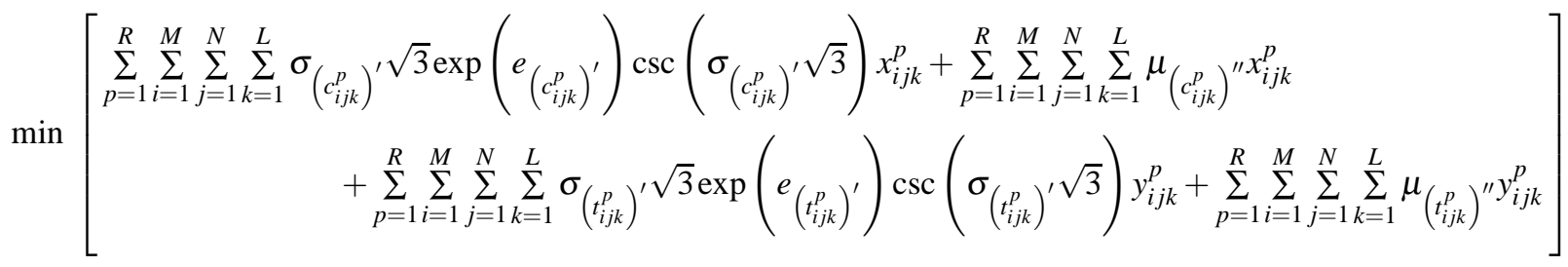

$$
\begin{aligned}
& \left\{\begin{array}{l}
\sum_{j=1}^{N} \sum_{k=1}^{L} x_{i j k}^{p} \leq \exp \left(e_{\left(a_{i}^{p}\right)^{\prime}}+\frac{\sqrt{3} \sigma_{\left(a_{i}^{p}\right)^{\prime}}}{\pi} \ln \frac{1-\alpha_{i}^{p}}{\alpha_{i}^{p}}\right), \forall i \in M ; \forall p \in R \\
\sum_{j=1}^{N} \sum_{k=1}^{L} x_{i j k}^{p} \leq \mu_{\left(a_{i}^{p}\right)^{\prime \prime}}+\sigma_{\left(a_{i}^{p}\right)^{\prime \prime}}\left(1-\alpha_{i}^{p}\right), \forall i \in M ; \forall p \in R \\
\sum_{i=1}^{M} \sum_{k=1}^{L} x_{i j k}^{p} \geq \exp \left(e_{\left(b_{i}^{p}\right)^{\prime}}+\frac{\sqrt{3} \sigma_{\left(b_{i}^{p}\right)^{\prime}}}{\pi} \ln \frac{\beta_{j}^{p}}{1-\beta_{j}^{p}}\right), \forall j \in N ; \forall p \in R \\
\sum_{i=1}^{M} \sum_{k=1}^{L} x_{i j k}^{p} \geq \mu_{\left(b_{i}^{p}\right)^{\prime \prime}}+\sigma_{\left(b_{i}^{p}\right)^{\prime \prime}} \beta_{j}^{p}, \forall j \in N ; \forall p \in R \\
\sum_{p=1}^{R} \sum_{i=1}^{M} \sum_{k=1}^{L} x_{i j k}^{p} \leq \exp \left(e_{\left(e_{k}\right)^{\prime}}+\frac{\sqrt{3} \sigma\left(e_{i}^{p}\right)^{\prime}}{\pi} \ln \frac{1-\gamma_{k}}{\gamma_{k}}\right), \forall k \in L \\
\sum_{p=1}^{R} \sum_{i=1}^{M} \sum_{k=1}^{L} x_{i j k}^{p} \leq \mu_{\left(e_{k}\right)^{\prime \prime}}+\sigma_{\left(e_{k}\right)^{\prime \prime}}\left(1-\gamma_{k}\right), \forall k \in L \\
x_{i j k}^{p} \geq 0, \forall i \in M ; \forall j \in N ; \forall k \in L ; \forall p \in R \\
y_{i j k}^{p}=\left\{\begin{array}{l}
1 \text { if } x_{i j k}^{p}>0 \\
0 \text { if } x_{i j k}^{p}=0
\end{array}, \forall i \in M ; \forall j \in N ; \forall k \in L ; \forall p \in R\right.
\end{array}\right.
\end{aligned}
$$

\section{Conclusions}

In this paper, uncertain random programming models for the fixed charge multi-item solid transportation problem is presented. Applying uncertainty and randomness, the model is transformed into its deterministic form. Then the deterministic model based on the expected value of each objective under the chance constraints is reduced to classical single objective programming problems. Also, fixed charge multi-item STP models for each uncertain random variable are illustrated with numerical examples. It is noted that uncertain random programming can be considered so widely in real-world applications since uncertain criteria broadly appear in all kinds of real-world mathematical programming problems.

\section{Competing interests}

The authors declare that they have no competing interests. 


\section{Authors' contributions}

All authors have contributed to all parts of the article. All authors read and approved the final manuscript.

\section{References}

[1] Shell, E. (1955). Distribution of a product by several properties, Directorate of Management Analysis. In Proceedings of the Second Symposium in Linear Programming (Vol. 2, pp. 615-642).

[2] Haley KB (1962) New methods in mathematical programming-The solid transportation problem. Operations Research 10(4): 448-463.

[3] Dalman H, Guzel, N and Sivri M (2016) A fuzzy set-based approach to multi-objective multi-item solid transportation problem under uncertainty. International Journal of Fuzzy Systems, 18(4), 716-729.

[4] Dalman H, Sivri M (2017) Multi-objective Solid Transportation Problem in Uncertain Environment. Iranian Journal of Science and Technology, Transactions A: Science, 41(2), 505-514.

[5] Das A, Bera UK and Maiti M (2016) A breakable multi-item multi stage solid transportation problem under budget with Gaussian type-2 fuzzy parameters. Applied Intelligence, 45(3), 923-951.

[6] Kundu P, Kar S, and Maiti M (2013) Multi-objective multi-item solid transportation problem in fuzzy environment. Applied Mathematical Modelling, 37(4), 2028-2038.

[7] Ojha A, Das B, Mondal S and Maiti M (2010). A stochastic discounted multi-objective solid transportation problem for breakable items using analytical hierarchy process. Applied Mathematical Modelling, 34(8), 2256-2271.

[8] Ojha A, Das B, Mondal S and Maiti M (2010) A solid transportation problem for an item with fixed charge, vechicle cost and price discounted varying charge using genetic algorithm. Applied Soft Computing, 10(1), 100-110.

[9] Liu B (2007) Uncertainty theory. In Uncertainty Theory (pp. 205-234). Springer, Berlin, Heidelberg.

[10] Liu B (2009) Some research problems in uncertainty theory. Journal of Uncertain Systems, 3(1), 3-10.

[11] Liu B (2010) Uncertain risk analysis and uncertain reliability analysis. Journal of Uncertain Systems, 4(3), 163-170.

[12] Liu B (2012) Why is there a need for uncertainty theory. Journal of Uncertain Systems, 6(1), 3-10.

[13] Chen X and Liu B (2010) Existence and uniqueness theorem for uncertain differential equations. Fuzzy Optimization and Decision Making, 9(1), 69-81.

[14] Liu B (2016) Some preliminary results about uncertain matrix. Journal of Uncertainty Analysis and Applications, 4(1), 11.

[15] Yao K and Liu B (2017) Uncertain regression analysis: An approach for imprecise observations. Soft Computing, 1-4.

[16] Bai X, Gao J and Liu Y (2017) Prepositioning emergency supplies under uncertainty: a parametric optimization method. Engineering Optimization, 1-20.

[17] Zhang B and Peng J (2013) Uncertain programming model for uncertain optimal assignment problem. Applied Mathematical Modelling, 37(9), 6458-6468.

[18] Liu B, and Yao K (2015) Uncertain multilevel programming: algorithm and applications. Computers and Industrial Engineering, 89, 235-240.

[19] Cui Q, and Sheng Y (2013). Uncertain programming model for solid transportation problem. International Information Institute (Tokyo). Information, 16(2), 1207.

[20] Zhang B, Peng J, Li S, Chen L (2016) Fixed charge solid transportation problem in uncertain environment and its algorithm. Computers and Industrial Engineering, 102, 186-197.

[21] Gao Y, Yang L and Li S (2016) Uncertain models on railway transportation planning problem. Applied Mathematical Modelling, 40(7), 4921-4934.

[22] Dalman H (2016) Uncertain programming model for multi-item solid transportation problem. International Journal of Machine Learning and Cybernetics, in press, doi: 10.1007/s13042-016-0538-7. 
[23] Liu B (2009) Theory and practice of uncertain programming (Vol. 239). Berlin: Springer.

[24] Liu, Y. (2013). Uncertain random variables: a mixture of uncertainty and randomness. Soft Computing, 17(4), 625-634.

[25] Liu, Y. (2013). Uncertain random programming with applications. Fuzzy Optimization and Decision Making, 1-17.

[26] Liu, B. (2014). Uncertain random graph and uncertain random network. Journal of Uncertain Systems, 8(1), 3-12.

[27] Wen M and Kang R (2016) Reliability analysis in uncertain random system. Fuzzy Optimization and Decision Making, 15(4), 491-506.

[28] Yao K and Gao J (2016) Law of large numbers for uncertain random variables. IEEE Transactions on Fuzzy Systems, 24(3), 615-621.

[29] Gao R and Ralescu DA (2017) Convergence in Distribution for Uncertain Random Variables. IEEE Transactions on Fuzzy Systems. [30] Sheng Y and Gao Y (2016) Shortest path problem of uncertain random network. Computers and Industrial Engineering, 99, 97-105. [31] Li B (2011) Uncertainty theory: A branch of mathematics for modeling human uncertainty. Springer-Verlag, Berlin, 13, 3-20.

[32] Liu YH and Ha M (2010) Expected value of function of uncertain variables. Journal of uncertain Systems, 4(3), 181-186. 